\title{
Correlation between extent of sinus surgery, radiographic disease, and postoperative outcomes*
}

\author{
Noel Ayoub1, Evan Walgama',2, Andrew Thamboo 1,3, Wirach \\ Chitsuthipakorn ${ }^{4}$, Zara M. Patel', Jayakar V. Nayak', Peter H. Hwang' \\ ' Department of Otolaryngology - Head and Neck Surgery, Stanford University, Palo Alto, CA, United States \\ 2 Department of Otolaryngology - Head and Neck Surgery, Cedars-Sinai Medical Center, Los Angeles, CA, United States \\ ${ }^{3}$ Department of Otolaryngology - Head and Neck Surgery, University of British Columbia, Vancouver, BC, Canada \\ ${ }^{4}$ Center of Excellence in Otolaryngology, Head \& Neck Surgery. Rajavithi Hospital, Rangsit University. Bangkok, Thailand
}

Rhinology 58: 1, $36-44,2020$

https://doi.org/10.4193/Rhin19.213

*Received for publication:

June 8, 2019

Accepted: July 15, 2019

\begin{abstract}
Background: The extent of endoscopic sinus surgery (ESS) required for optimal outcomes in chronic rhinosinusitis (CRS) is undefined. We evaluated whether concordance between the extent of surgery and degree of radiographic disease influences postoperative outcomes.
\end{abstract}

Methods: 247 CRS patients who underwent ESS were retrospectively assigned a concordance score reflecting the similarity between the extent of surgery and degree of radiographic disease. 0 points were assigned when sinusotomy was performed on a diseased sinus, or no sinusotomy was performed on a nondiseased sinus; +1 for sinusotomy on a nondiseased sinus; and -1 for a diseased sinus left unopened. The total possible score ranged from -10 to +10 . Patients were divided into 5 subgroups according to variance from complete concordance. SNOT-22 scores and revision rates were compared at 6 and 24 months.

Results: All five subgroups had similar preoperative SNOT-22 scores and improved at 6 months postoperatively. At 6 months postoperatively, the most conservatively operated and most extensively operated subgroups each achieved equivalent improvements in SNOT-22 as the completely concordant subgroup. At 24 months, the most extensively operated subgroup had a 12.5-point smaller improvement in SNOT-22 scores compared to the completely concordant subgroup. Multivariate analysis showed no association between concordance score and revision rate.

Conclusions: Symptom improvement and revision rates after ESS do not appear to correlate with the degree of concordance between extent of surgery and radiographic disease. More extensive surgery than indicated by CT confers neither greater symptomatic improvement nor long-term detriment.

Key words: Paranasal sinuses, sinusitis, endoscopic sinus surgery, chronic rhinosinusitis, patient reported outcome measures, quality of life

\section{Introduction}

Endoscopic sinus surgery (ESS) is now one of the 10 most common ambulatory surgeries performed in the US each year ${ }^{(1,2)}$. More advanced endoscopic techniques, improved surgical instrumentation, increased patient acceptance of sinus surgery, and a greater number of fellowship-trained rhinologists have increased the frequency and scope of sinus surgeries performed. From 2000 to 2009, for example, the frequency of frontal sinus procedures doubled, and operations involving all sinuses tripled $^{(3)}$.
While efforts to define appropriateness criteria for ESS have recently been published, the indications for, and timing of surgical intervention for recalcitrant CRS continue to be a subject of considerable debate ${ }^{(4-6)}$. Furthermore, determining the appropriate extent of surgery for any given patient also remains an unresolved issue. Surgeons must decide whether it is preferable to perform a conservative surgery that treats only the diseased sinuses identified on preoperative imaging (or fewer), or a comprehensive surgery that may extend beyond the areas 
of radiographic disease. A conservative approach may preserve anatomy and physiology of non-diseased tissues, minimise the risks of iatrogenic injury, and require less operative time. (4) In some cases, sinus mucosal thickening may resolve with the surgical ventilation of adjacent sinuses without actually opening the diseased sinus itself ${ }^{(7,8)}$. Conversely, a more extensive approach that extends surgery beyond the diseased sinuses to adjacent non-diseased sinuses may preempt the need for additional future surgery if non-diseased sinuses were to become diseased in the future. Other rationales for performing surgery beyond the extent of known disease include the identification of surgical landmarks within non-diseased sinuses to facilitate safe dissection of diseased sinuses, and the enhancement of delivery of topical therapies to the sinuses ${ }^{(9)}$.

Chronic rhinosinusitis is increasingly recognised as an aggregate of heterogeneous phenotypes, and it is this variability in disease patterns that renders it difficult to standardise the surgical approach for each patient. Deciding the appropriate amount of sinus surgery requires a balanced consideration of patientspecific and disease-specific factors to maximise the likelihood of symptom relief, while minimising surgical risk, morbidity and cost. We sought to compare symptomatic outcomes and surgical revision rates based on the degree of concordance between the extent of surgery and extent of radiologic disease in ESS.

\section{Materials and methods}

Patient selection

Approval for this retrospective analysis was obtained from the Stanford University Institutional Review Board. The Stanford Translational Research Integrated Database Environment (STRIDE) was utilised to identify eligible patients ${ }^{(10)}$. The database was searched for International Classification of Diseases (ICD-9) code 473.x for chronic sinusitis and one or more of the following Current Procedural Terminology (CPT) codes recorded during an outpatient surgical procedure: maxillary antrostomy (31256 or 3126), anterior ethmoidectomy (31254), anterior and posterior ethmoidectomy (31255), frontal sinusotomy (31276), or sphenoidotomy (31287 or 31288). Electronic medical records of patients who fit the inclusion criteria were reviewed, and data was extracted and stored on REDCap, a secure web-based application ${ }^{(11)}$.

All patients in the study underwent primary or revision sinus surgery at the Stanford Sinus Center by one of two fellowshiptrained rhinologists (PHH or JVN) between January 1, 2010 and December 31, 2013. Patients were deemed eligible surgical candidates if they failed appropriate medical therapy, which at our clinic most typically includes a combination of oral antibiotics, topical steroids and saline irrigations. Inclusion criteria included age greater than 18 at time of surgery and a diagnosis of chronic rhinosinusitis based on consensus criteria proposed by the American Academy of Otolaryngology-Head and Neck Surgery (AAOHNS) 2015 Clinical Practice Guidelines ${ }^{(12)}$. Patients were excluded from the study if the pre-operative computed tomography (CT) scan could not be located, preoperative SNOT-22 questionnaires were incomplete, or postoperative follow-up was less than 6 months. Patients undergoing ESS for recurrent acute sinusitis, fungus balls, or isolated sinus mucoceles were also excluded.

\section{Data extraction}

Patient demographics and relevant clinical data were collected, including age, gender, prior sinus surgery, and presence of nasal polyposis. Comorbidities were recorded, including a history of asthma, aspirin sensitivity, atopy verified by skin prick or radioallergosorbent test, obstructive sleep apnea, immunodeficiency, diabetes, depression, and current or prior tobacco use. Past medical and social histories were extracted based on a review of medical records and patient-reported medical history.

The extent of sinus surgery performed was derived from operative notes in the medical record to determine whether or not the following procedures had been performed: maxillary antrostomy, anterior and/or posterior ethmoidectomy, sphenoid sinusotomy, and frontal sinusotomy. Using the Centricity Enterprise Web ${ }^{\mathrm{TM}}$ software (GE Medical Systems Informational Technologies, Waukesha, WI, USA), the Lund-Mackay (LM) score was calculated from the preoperative CT scan. The LM scoring system is a validated categorical scale used to define the extent of sinonasal disease on $\mathrm{CT}^{(13,14)}$. Each sinus was blindly scored by two reviewers, with a third reviewer serving as the tiebreaker, when needed. The bilateral maxillary, anterior ethmoid, posterior ethmoid, sphenoid, and frontal sinuses were scored from 0-2, with 0 correlating to a normal sinus, 1 to partial opacification or mucosal thickening, and 2 to a fully opacified sinus. The bilateral ostiomeatal complexes were scored 0 if patent or 2 if obstructed. The total possible LM score ranged from 0 to 24, with 24 representing the most extensive disease.

\section{Defining the concordance score}

To characterise overall surgical philosophy, a concordance scoring system was developed to correlate the sinusotomies performed with the extent of disease based on preoperative imaging (Figure 1). A concordance score of $-1,0$, or +1 was assigned to each of five sinuses bilaterally (maxillary, anterior ethmoid, posterior ethmoid, sphenoid, and frontal), regardless of whether it had been operated on or not. A concordance score of 0 was assigned when imaging and surgery were concordant, i.e. a sinusotomy was performed on a diseased sinus (Lund-Mackay 1 or 2), or no sinusotomy was performed on a nondiseased sinus (Lund-Mackay 0). A score of -1 was assigned when a diseased sinus was left intact (more conservative approach), and +1 was 

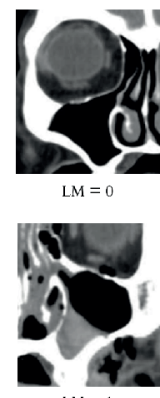
$\mathrm{LM}=$

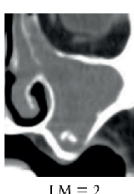

$\mathrm{M}=2$

\section{.}

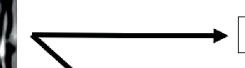

Sinusotomy

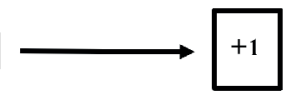

,
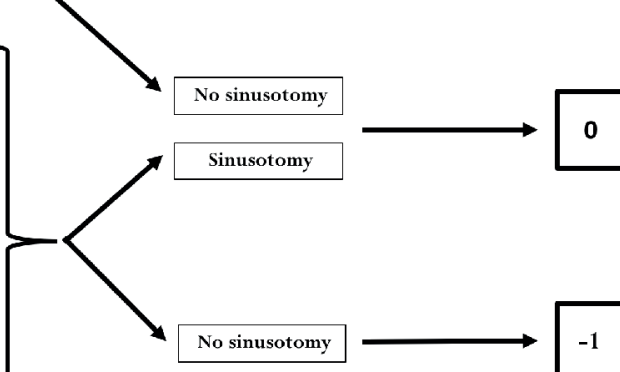

assigned when a sinusotomy was performed on a nondiseased sinus (more extensive approach). The scores were summed for a total possible score ranging from -10 to +10 . A total concordance score of 0 implied that all nondiseased sinuses were left surgically unopened and all diseased sinuses underwent sinusotomy. Similarly, a total concordance score of +10 indicated that sinusotomies were performed on every sinus, each being radiographically normal. Patients who were treated with a mixed surgical philosophy, in which some non-diseased sinuses were opened (concordance score +1 ) whereas other diseased sinuses were not opened (concordance score -1), were excluded to limit score neutralisation.

\section{Outcomes}

The twenty-two item Sinonasal-Outcome Test (SNOT-22) was administered at the preoperative visit and during routine follow up 6 and 24 months postoperatively. The SNOT-22 is a validated, disease-specific questionnaire in which patients report the presence and severity of 22 commonly experienced symptoms and social consequences of chronic rhinosinusitis over the prior 2-week period ${ }^{(15)}$. Each question scales from 0 to 5, with 0 indicating "no problem" and 5 "problem as bad as it can be." The highest possible score is 110 , corresponding to the most severe symptoms. The mean score in patients without sinonasal disease is 9.3 (95\% Cl 7.5-11.1), and an 8.9-point difference following intervention is deemed clinically significant ${ }^{(15)}$. The incidence of additional revision sinus surgery performed during the study period was also recorded at 6 months and 24 months.

\section{Statistical analysis}

Statistical analysis was performed using SAS 9.4 (SAS Institute, Inc, Cary, NC, USA). The prevalence of baseline characteristics, comorbidities, and prior sinus surgery was compared across treatment subgroups using Pearson Chi square ( $x 2$ ) testing,

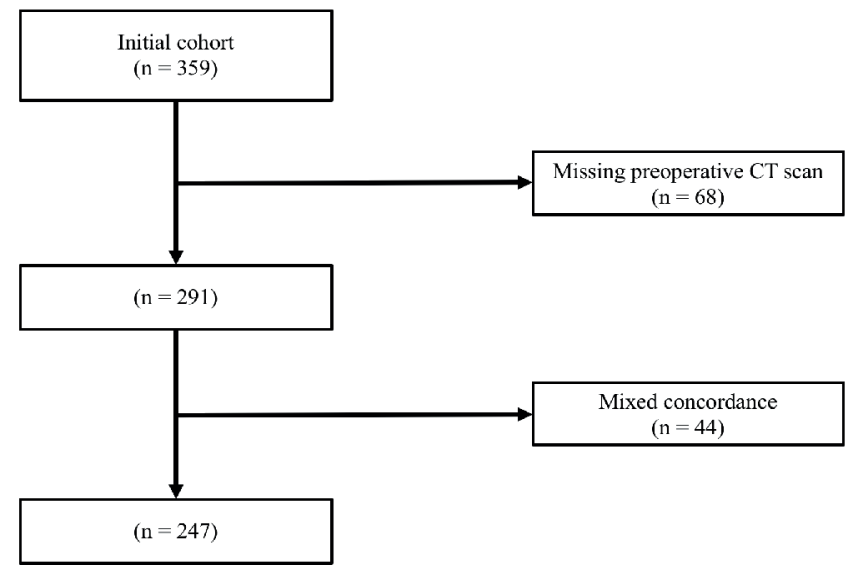

Figure 2. Determination of study cohort.

t-tests, and 1-way analysis of variance (ANOVA), where appropriate. Symptom score and LM score were compared using Wilcoxon rank-sum test across treatment subgroups at baseline, 6 months, and 24 months. The quantitative change in symptom score and LM score from baseline to 6 months and 24 months were compared across treatment cohorts using paired t tests. Associations between treatment score and symptom score were examined using robust linear regression models with Huber's M-estimation, controlling for patient's baseline characteristics. Stratified models were also built to examine whether the associations varied by LM-score and comorbidities. Logistic regression was used to evaluate the association between baseline characteristics and outcomes of ESS. A standard alpha level 0.05 was used to determine significance for all calculations.

\section{Results}

Demographics and baseline characteristics of entire population

Three hundred and fifty-nine patients met inclusion criteria (Figure 2). Of these, 68 were excluded due to lack of availability of preoperative $\mathrm{CT}$ imaging in the electronic medical records and hospital media library. Forty-four additional patients with mixed concordances (see Methods) were also excluded. This left 247 patients for the analysis, whose baseline clinical characteristics are noted in Table 1: 112 (45.3\%) were female with an average age of 47.1 (range 19 to 87 ) at time of surgery. 135 (54.7\%) had one or more prior sinus surgeries, $118(47.7 \%)$ had nasal polyposis, 23 (9.3\%) had ASA sensitivity, and 25 (10.1\%) had cystic fibrosis. The total mean preoperative LM and SNOT-22 scores were $9.4 \pm 5.1$ (range 0 to 20 ) and $47.2 \pm 22.3$ (range 3 to 101), respectively, with no significant correlation between the two (Figure 3). 163 (66\%) of patients had two-year follow up data available, with a similar rate of subjects lost to follow up across all subgroups $(p=0.85)$. The average time from preoperative CT scan to surgery was $2.0 \pm 2.9$ months. 


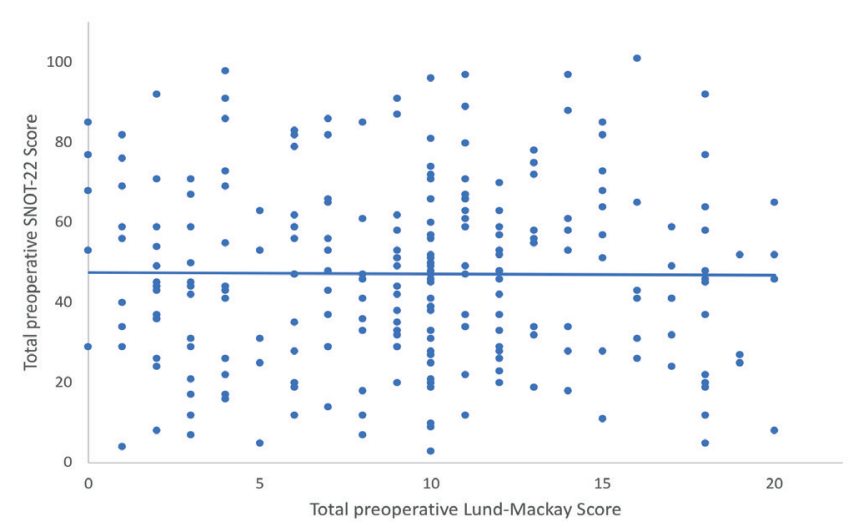

Figure 3. Association between Preoperative Lund-Mackay and SNOT-22 scores for cohort of 247 patients.

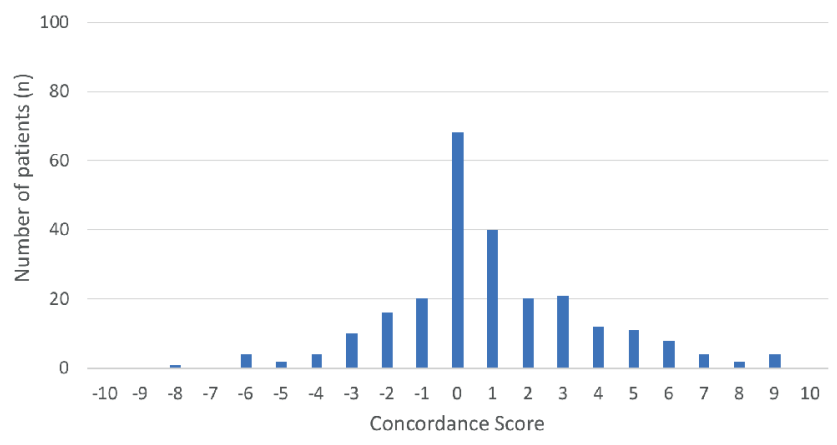

Figure 4. Distribution of patients by concordance score.

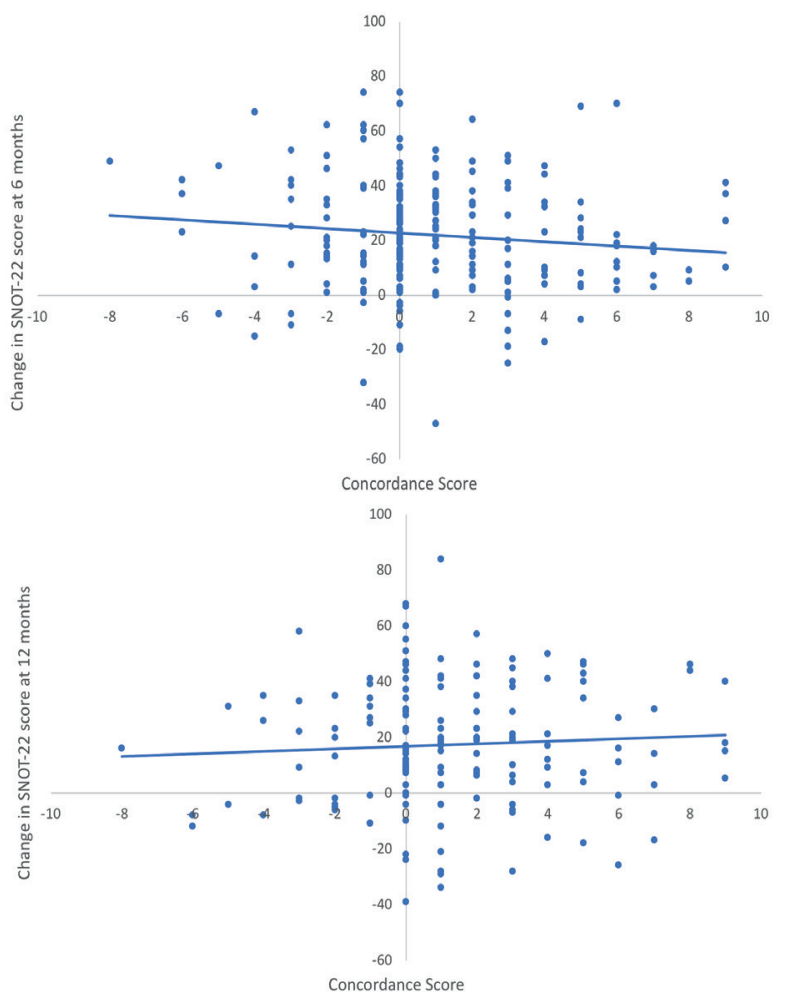

Figure 5. A) Correlation between concordance score and postoperative change in SNOT-22 scores at 6 months. B) Correlation between concordance score and postoperative change in SNOT-22 scores at 24 months.

\section{Concordance scores}

Sixty-eight (27.5\%) of the total 247 patients underwent ESS that exactly correlated with the extent of disease observed on preoperative imaging (concordance score $=0$ ); 57 (23.0\%) had more conservative surgery (concordance score $<0$ ); and 122 (49.4\%) patients had more extensive surgery (concordance score $>0$ ) (Figure 4). Overall concordance scores ranged from -8 to +9 with a median of 0 and mean of $0.9 \pm 2.8$. The maxillary sinus exhibited the highest incidence of radiographic disease on preoperative imaging (84.8\%) and sphenoid sinus the lowest (61.9\%). Of the sinuses with a Lund-Mackay subscore of 0 , the maxillary sinus was the most frequently operated upon (72.1\%), and the sphenoid (33.5\%) and frontal (33.0\%) sinuses the least. Similarly, of the sinuses with a Lund-Mackay score of 1 or 2, the maxillary sinus again underwent sinusotomy (97.1\%) most frequently, in contrast to the sphenoid (90.5\%) and frontal (89.0\%) sinuses, which had the lowest rates of sinusotomy.

\section{Demographics and baseline characteristics by subgroup} Patients were divided into 5 subgroups based on concordance score: $\leq-3$ (21 patients), -2 to -1 (36 patients), 0 (68 patients), +1 to +2 ( 60 patients), and $\geq+3$ (62 patients) (Table 1$)$. All subgroups had a similar incidence of cystic fibrosis $(p=0.34)$, depression ( $p=0.66)$, and smoking $(p=0.14)$. When compared to the other subgroups, the completely concordant subgroup (sco$\mathrm{re}=0$ ) had the highest absolute preoperative Lund-Mackay score (mean 12, p<0.001). Given the dependence of the concordance score on pre-operative Lund-Mackay score, total Lund-Mackay score was lowest in the most extensively treated subgroup (concordance score $\geq 3, \mathrm{p}<0.001$ ).

\section{Comparison of absolute SNOT-22 scores and postoperative changes in score}

Table 2 details the absolute pre- and postoperative SNOT-22 scores, as well as the change in scores, throughout the study period. The completely concordant subgroup (score $=0$ ) had the highest baseline SNOT-22 score (mean 48), although no statistically significant difference in baseline preoperative SNOT-22 scores was observed between the five subgroups $(p=0.198)$. Absolute SNOT-22 scores were similar across all subgroups at 6 months $(p=0.800)$ but not at 24 months $(p=0.014)$.

Collectively, the mean total SNOT-22 scores for the entire cohort decreased from 47 preoperatively to 22 six months after surgery $(p<0.0001)$ and remained improved at the end of the 2-year follow up period with a score of $26(p<0.0001)$. Compared to their own baseline scores, patients in every subgroup demonstrated clinically and statistically significant improvements in SNOT-22 
Table 1. Baseline characteristics by concordance subgroup.

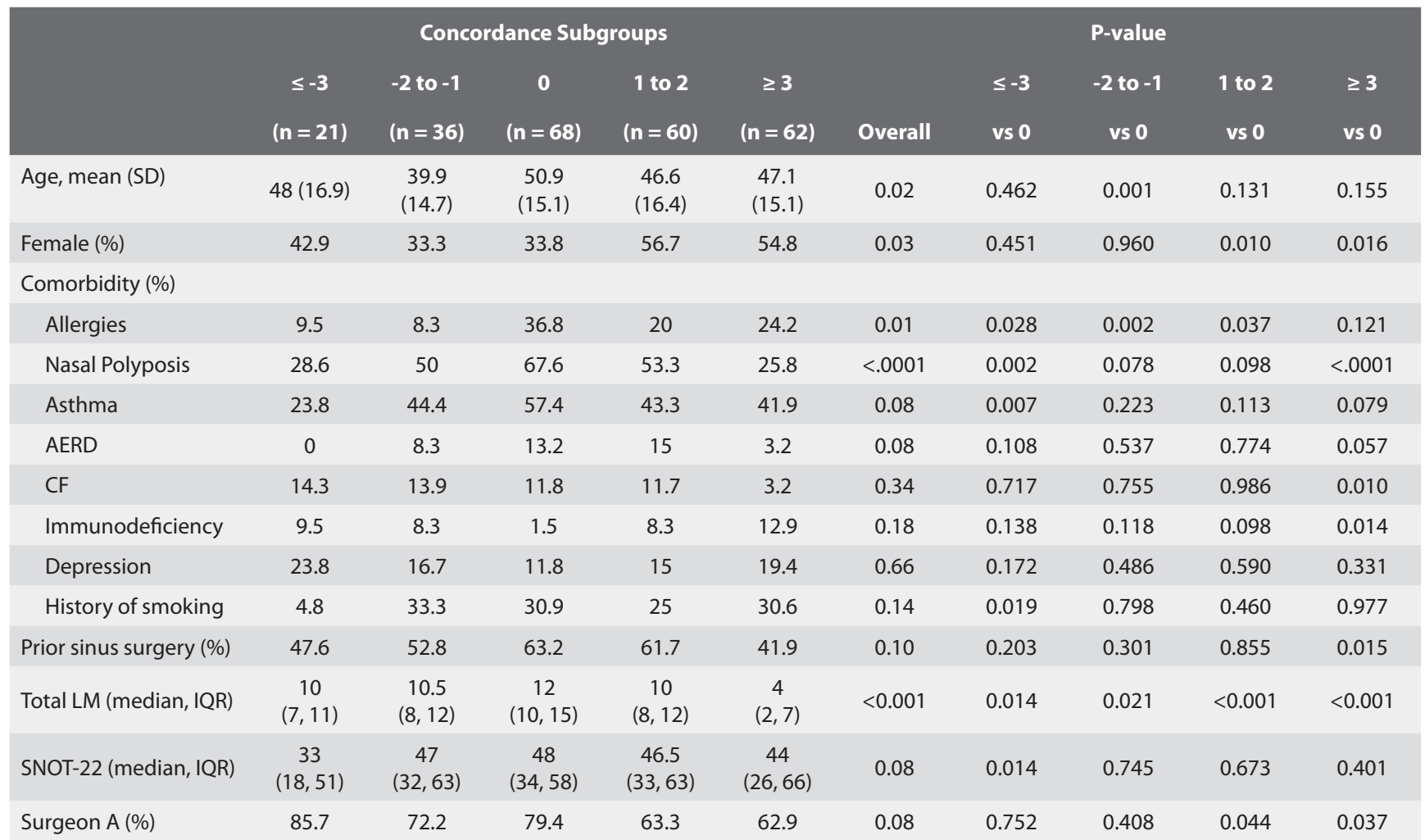

Table 2. Postoperative SNOT-22 scores within each concordance subgroup.

\begin{tabular}{|c|c|c|c|c|c|c|c|}
\hline & \multicolumn{7}{|c|}{ Concordance score } \\
\hline & $\leq-3$ & -2 to -1 & 0 & 1 to 2 & $\geq 3$ & All & P-value \\
\hline Baseline, median (IQR*) & $33(18,51)$ & $47(32,63)$ & $48(34,58.5)$ & $47(34,64)$ & $44(26,66)$ & $47(29,62)$ & 0.198 \\
\hline 6 months & $17(7,35)$ & $24(11,39)$ & $22(6,40)$ & $20(10.5,36)$ & $24(10,37)$ & $22(9,37)$ & 0.800 \\
\hline 24 months & $24(15,39)$ & $38(14,49)$ & $27(10,39)$ & $21(8,30)$ & $36(20,59)$ & $26(12,40)$ & 0.014 \\
\hline$\Delta$ Pre-op vs. 6 months ${ }^{* *}$ & $-15(-23,-5)$ & $-24(-33,-9)$ & $-26(-37,-12)$ & $-22(-37,-6)$ & $-18(-36,5)$ & $-20.5(-36,-8)$ & 0.465 \\
\hline$\Delta$ Pre-op vs. 24 months** & $-9(-21.5,-1)$ & $-15(-23,4)$ & $-22(-38,-9)$ & $-21(-41,-7)$ & $-9(-29,4)$ & $-16(-34,-3)$ & 0.037 \\
\hline MCID*** \% (6 months) & 62 & 77 & 81 & 74 & 69 & 75 & 0.416 \\
\hline MCID \% (24 months) & 50 & 67 & 74 & 63 & 62 & 65 & 0.474 \\
\hline
\end{tabular}

scores at 6 months (mean 22.0-point improvement, $\mathrm{p}<0.001$ ) and 24 months (mean 17.2-point improvement, $p<0.001$ ) after surgery.

When correlating changes in SNOT-22 scores with concordance score as a linear function, no difference in the change in SNOT22 scores was observed between the subgroups at any time point. (Figure 5) At 6 months postoperatively, patients treated with completely concordant surgical approaches achieved similar improvements in median SNOT-22 scores (26-point decrease) as those who underwent the most extensive (18-point decrease) and most conservative (15-point decrease) surgical approaches ( $p=0.465$ ) (Table 2). After 24 months, however, patients in the completely concordant subgroup demonstrated greater improvements in SNOT-22 scores (22-point improvement) than patients in both the most extensive (9-point improvement) and most conservative (9-point improvement) surgical subgroups $(p=0.037)$. Robust logistic regression using the completely concordant cohort (concordance score $=0$ ) as a reference group showed a 12.5-point greater decrease in SNOT-22 scores in the completely concordant subgroup compared to the most extensive subgroup at 24 months, a difference that was statistically significant $(p=0.003)$. No other statistically significant findings were observed.

Additional sinus surgeries 
Table 3. Revision rate at 6 and 24 months by concordance subgroups.

\begin{tabular}{|ccccccccc} 
& \multicolumn{4}{c}{ Concordance score } \\
& $\leq-3$ & -2 to -1 & 0 & 1 to 2 & $\geq 3$ & Total & P-value \\
\hline 6 months, $n(\%)$ & $0(0)$ & $0(0)$ & $2(2.9)$ & $5(8.3)$ & $2(3.2)$ & $9(3.6)$ & 0.175 \\
\hline 24 months, $n(\%)$ & $1(4.8)$ & $3(8.3)$ & $8(11.8)$ & $11(18.3)$ & $5(8.1)$ & $28(11.3)$ & 0.598 \\
\hline
\end{tabular}

The overall rate of revision sinus surgeries performed after 6 and 24 months postoperatively was 3.6 and $11.3 \%$, respectively (Table 3). The highest rate of revision sinus surgery was seen in the moderately extensive subgroup (concordance score 1 to 2 at 6 months (8.3\%) and at 24 months (18.3\%). Of the 68 patients who were treated with a completely concordant surgical approach, 2 (2.9\%) required additional procedures within 6 months, and an additional 6 between 12 and 24 months, for a total of 8 (11.8\%) overall. Despite these findings, no statistically significant differences were observed in the rates of sinus surgery at either time point among any of the concordance subgroups (all $p$-values $>0.05$ ). Further analysis of the data revealed a difference in revision rates between the two surgeons ( $1.7 \%$ vs. $20.8 \%, p<0.0001)$. Subsequent logistic regression controlling for surgeon, patient age, gender, Lund-Mackay score, and comorbidities showed no association between the rate of revision and concordance score (Table 4). Factoring surgeon into the multivariate analysis thus confirmed that there was no difference in revision rate by surgical approach.

\section{Discussion}

ESS is clearly beneficial in the management of recalcitrant rhinosinusitis, but the extent of surgery required to achieve clinical improvement is a judgment left to the individual surgeon ${ }^{(4,16)}$. The present study sought to identify whether different surgical philosophies regarding extent of surgery affect symptomatic outcomes or revision rates of sinus surgery. Viewed as a whole, our cohort showed statistically and clinically significant improvements in SNOT-22 scores after ESS throughout the 24-month follow up period, consistent with prior published data ${ }^{(17,18)}$. Notably, the degree of concordance between extent of surgery and radiologic disease had no clear impact on surgical outcome. Whether the extent of surgery was less than, equal to, or greater than the radiologic extent of disease, there was no trend in postoperative QOL improvement or revision rates. The major significant finding was a greater decrease in SNOT-22 scores after two years in surgeries that adhered to a completely concordant approach compared to those where the extent of surgery exceeded the extent of radiologic disease. It is possible that the large interquartile ranges and possibly underpowered nature of the study precluded more significant findings.

Arguments for pursuing a more extensive surgical approach
Table 4. Multivariate analysis of revision rate at 24 months.

\begin{tabular}{|c|c|c|c|}
\hline Variable & Odds ratio & $\mathrm{Cl}$ & p-value \\
\hline Surgeon & 0.106 & $0.038,0.298$ & $<.0001$ \\
\hline LM score & 1.037 & $0.905,1.188$ & 0.601 \\
\hline Age & 0.982 & $0.951,1.014$ & 0.267 \\
\hline Gender & 0.449 & $0.173,1.165$ & 0.099 \\
\hline Allergies & 0.981 & $0.307,3.135$ & 0.974 \\
\hline Polyps & 0.966 & $0.319,2.923$ & 0.951 \\
\hline Asthma & 1.465 & $0.516,4.155$ & 0.473 \\
\hline ASA sensitivity & 0.354 & $0.056,2.250$ & 0.271 \\
\hline Cystic fibrosis & 0.405 & $0.075,2.203$ & 0.296 \\
\hline Immunodeficiency & 3.081 & $0.669,14.203$ & 0.149 \\
\hline Depression & 0.346 & $0.070,1.707$ & 0.192 \\
\hline History of smoking & 0.37 & $0.106,1.288$ & 0.118 \\
\hline \multicolumn{4}{|l|}{ Concordance score } \\
\hline$\leq-3$ & 0.294 & $0.028,3.025$ & 0.303 \\
\hline-2 to -1 & 0.473 & $0.097,2.305$ & 0.354 \\
\hline 1 to 2 & 0.914 & $0.265,3.145$ & 0.886 \\
\hline$\geq 3$ & 0.28 & $0.056,1.399$ & 0.120 \\
\hline
\end{tabular}

than indicated by CT scan may include the desire to optimise distribution of topical medications, to improve access to sinuses in clinic, or to prevent possible progression of disease to healthy sinuses by preemptively opening them ${ }^{(9)}$. Opening nondiseased sinuses may also aid in the identification of endoscopic landmarks for surgical dissection, or there may be intraoperative findings such as mucus recirculation that may not be readily evident on a CT scan. Significant symptom burden might also sway a surgeon to operate more extensively; however, in our series, greater symptom severity was not associated with more extensive surgery, nor was it associated with a greater degree of postoperative improvement. An additional factor that might predict more extensive surgery could be a patient's history of prior sinus surgeries, or the presence of comorbidities such as AERD or cystic fibrosis with higher relapse rates of sinusitis ${ }^{(19,20)}$. Some surgeons believe that a more extensive procedure may prevent or delay the need for revision surgery. Our analysis, however, failed to show a reduction in the rate of reoperation after more extensive surgery compared to completely concordant or more conservative surgery. Similar to the decision to open greater or fewer sinuses, however, the decision to perform revision surgery incorporates a number of factors, including 
surgeon judgment.

A prospective, multi-institutional, observational study published in 2015 found a statistically, but not clinically, significant improvement in QOL scores 13 months following "complete" surgery (9). The authors defined "complete" surgery as performing bilateral frontal sinusotomy, ethmoidectomy, maxillary antrostomy, and sphenoidectomy; anything less was considered a "targeted" approach. While there was a statistical benefit to performing more extensive surgery, the practice of "complete" surgery did not clearly translate to symptom improvement or decreased rates of revision surgeries.

One of the arguments for pursuing a more conservative extent of surgery than indicated by CT is that a more minimal approach may be sufficient to alleviate patient's symptoms. In select patients with CRS with nasal polyps, for example, simple officebased polypectomy was shown to have an equivalent QOL benefit as formal endoscopic sinus surgery ${ }^{(21)}$. Less extensive surgery may also mitigate the risk of iatrogenic disease that might result from mucosal stripping, altered nasal nitric oxide concentrations, damaged ciliary function, or postoperative scarring resulting from surgery on a normal sinus ${ }^{(22)}$. Furthermore, a radiologically diseased sinus may improve simply by opening an adjacent diseased sinus ${ }^{(4,7)}$. A prior study has shown that in patients with frontal sinusitis, an anterior ethmoidectomy alone led to resolution of frontal sinus mucosal thickening in $88.5 \%$ of patients ${ }^{(23)}$. In another prospective study of surgical treatment for chronic frontal sinusitis, anterior ethmoidectomy alone resulted in similar postoperative QOL outcomes compared to anterior ethmoidectomy performed with frontal sinusotomy ${ }^{(7)}$. Some proponents of less extensive surgery have suggested that surgically opening a normal sinus may predispose it to become diseased if the severity of sinusitis progresses ${ }^{(24)}$. In a study of revision surgery, Bhattacharyya observed an increase (trend but not statistically significant) in the incidence of sinusitis in previously nondiseased sinuses following sinusotomy compared to nondiseased sinuses that were left intact ${ }^{(25)}$.

Subjective complaints and symptom burden initially drive a patient to seek medical attention for CRS, and CT scans are an important determinant of the need for surgery; yet numerous studies have demonstrated a lack of correlation between patient symptoms and radiographic disease ${ }^{(26-28)}$. While one might expect a completely opacified sinus to cause more severe symptoms than partial opacification, one study found no association between degree of opacification and severity of symptoms (29). Thus, the surgeon often faces a conundrum of whether to rely more on symptomatic or radiologic guidance for determining extent of surgery. It remains an individualised, subjective decision for the surgeon whether to conform to the extent of radiographic disease, versus operating less than or more than the CT scan indicates. And even the degree to which a sinus is opened may not necessarily affect outcome. For example, a study comparing wide maxillary antrostomy versus uncinectomy without disturbance of the maxillary ostium showed comparable improvements in LM and quality of life scores between the two groups ${ }^{(30)}$.

The interplay between anatomic obstruction and inflammatory etiologies contributing to CRS can also be difficult to discern in determining the optimal extent of surgery. Patients who continue on postoperative medical therapy may experience good control of symptoms despite not having had all affected sinuses opened. In our study, 57 patients (concordance score $<0$ ) underwent surgery that did not directly treat all the radiographically diseased sinuses, yet they demonstrated comparable postoperative outcomes to the 190 who had surgery that conformed to or slightly exceeded the radiographic disease.

In the planning of endoscopic sinus surgery, the decision to perform each sinusotomy requires a proper risk-benefit analysis. Our study showed that selecting a less extensive surgical approach relative to radiologic distribution of disease may yield similar outcomes as a completely concordant approach. In addition, we found no clear benefit of extending the surgery beyond the diseased sinuses identified on preoperative imaging. But as a corollary to this, when performed properly, more extensive surgery also did not appear to lead to worse postoperative outcomes at 6 months. No overarching recommendation can thus be made to always undertreat or over-treat the sinuses, or to perform a surgery that exactly conforms to the disease dictated by preoperative imaging. Rather than abiding by a single surgical philosophy, a surgeon should develop a patient-specific rationale for the extent of surgery for any given patient, incorporating surgeon judgment, endoscopic evidence of disease, preoperative imaging, and symptom burden. The surgical plan must then be executed with meticulous surgical technique and appropriate postoperative medical management. Irrespective of the approach selected, performing ESS necessitates appropriate patient selection based on symptom burden and prior medical therapy, superior surgical technique, and adequate postoperative care.

There are inherent limitations of our study, as with any retrospective study. Specific medication usage and compliance with medical regimens could not be tracked adequately, preventing inclusion of medication usage as a variable for analysis. The $34 \%$ rate of follow-up loss at the 2-year postoperative mark may limit the generalisability of conclusions that can be made regarding long-term SNOT-22 and revision rate outcomes. However, given that the 2-year follow-up capture rate was statistically comparable across all subgroups studies, we believe the potential impact 
of follow-up attrition was distributed evenly across all groups. It is also possible that a longer follow-up period might have revealed a differentiation in outcomes that was not detectable within our follow-up period.

The global concordance score developed for this study prevented determination of whether surgical treatment or nontreatment of specific sinuses (e.g., frontal versus sphenoid) differentially influenced postoperative outcomes. Given the lack of a validated scoring system to assess extent, the concordance score was used as a surrogate measure for the degree of surgery relative to radiographic findings. Additionally, one component of the concordance score is the LM score, which does not differentiate between a sinus with score of 1 that is nearly fully opacified (e.g. 80-99\%) or minimally opacified (e.g. 0-10\%).

This study design also did not allow for a clear discernment of the reasons that led to more or less extensive surgery for each patient, which may have included surgeon training or experience, patient preference, overall risk assessment, or disease severity. Patients with more severe underlying co-morbidities such as AERD and cystic fibrosis might have been expected to foster a bias towards opening non-diseased sinuses (i.e., skewing towards higher concordance scores). However, in actuality there were no significant differences in the distribution of concordance scores in patients with AERD or CF compared to those patients without. This suggests that the selection of surgical strategy encompasses more complexity beyond the presence of associated co-morbidities that might predict more severe disease.

Similarly, we were not able to ascertain all the factors leading to the decision for revision surgery for any given patient. Thus, the notable difference in revision rates observed between surgeons could not be explained within the scope of this study. A larger, prospective study tracking a greater number of surgeons could reduce the variance in revision rates between surgeons and provide more clarity regarding the impact of extent of surgery on revision rates.
Many distinct factors inform the decision-making process regarding surgical planning in ESS. The decision to perform each sinusotomy requires appropriate risk-benefit analysis and value judgments. The extent of ESS should ideally be fine-tuned to specific patient circumstances to form a logical plan and optimise outcomes. Generally speaking, more conservative approaches with respect to extent of surgery do not compromise the magnitude of postoperative improvement, nor do they lead to a greater rate of surgical revision. Conversely, more extensive surgery than indicated by CT scan does not confer greater symptomatic benefit nor reduce the need for later revision, nor does it cause worse postoperative outcomes. The outcomes presented in this study appear to be largely independent of the extent of surgery performed relative to radiographic burden of disease, suggesting that other factors play a role in determining postoperative quality of life outcomes and necessity of revision procedures.

\section{Acknowledgements}

There was no funding for this work.

\section{Authorship contribution}

NA: developed the research plan, data collection, contributed to data analysis and interpretation of the results, wrote the manuscript with input from all other authors. EW, AT, : developed the research plan, data collection, contributed to data analysis and interpretation of the results, contributed to the final version of the manuscript. ZMP: helped shape the research plan, contributed to data analysis and interpretation of the results, contributed to the final version of the manuscript. JVN: helped shape the research plan, contributed to data analysis and interpretation of the results, contributed to the final version of the manuscript. $\mathrm{PHH}$ : conceived the idea, developed the research plan, contributed to data analysis and interpretation of the results, contributed to the final version of the manuscript, supervised the project

\section{Conflict of interest}

None of the authors have any financial disclosures or conflicts of interest.

\section{References}

1. Bhattacharyya N. Ambulatory sinus and nasal surgery in the United States: demographics and perioperative outcomes. Laryngoscope 2010; 120:635-8.

2. Stammberger $\mathrm{H}$, Posawetz W. Functional endoscopic sinus surgery. Concept, indications and results of the Messerklinger technique. Eur Arch Otorhinolaryngol 1990; 247:63-76.

3. Pynnonen MA, Davis MM. Extent of sinus surgery, 2000 to 2009: a population-based study. Laryngoscope 2014; 124:820-5.
4. Orlandi RR, Kingdom TT, Hwang PH, et al. International Consensus Statement on Allergy and Rhinology: Rhinosinusitis. Int Forum Allergy Rhinol 2016; 6 Suppl 1:S22S209.

5. Rudmik L, Soler ZM, Hopkins C, et al Defining appropriateness criteria for endoscopic sinus surgery during management of uncomplicated adult chronic rhinosinusitis: a RAND/UCLA appropriateness study. Int Forum Allergy Rhinol 2016; 6:557-67.

6. Orlandi RR, Smith TL, Marple BF, et al. Update on evidence-based reviews with recommendations in adult chronic rhinosinusitis. Int Forum Allergy Rhinol 2014; 4 Suppl 1:S1-S15.

7. Abuzeid WM, Mace JC, Costa ML, et al. Outcomes of chronic frontal sinusitis treated with ethmoidectomy: a prospective study. Int Forum Allergy Rhinol 2016; 6:597-604.

8. Bikhazi N, Light J, Truitt T, et al. Standalone balloon dilation versus sinus surgery for chronic rhinosinusitis: a prospective, multicenter, randomized, controlled trial with 1-year follow-up. Am J Rhinol Allergy 2014; 
28:323-9.

9. DeConde AS, Suh JD, Mace JC, Alt JA, Smith TL. Outcomes of complete vs targeted approaches to endoscopic sinus surgery. Int Forum Allergy Rhinol 2015; 5:691-700.

10. Lowe HJ, Ferris TA, Hernandez PM, Weber SC. STRIDE--An integrated standards-based translational research informatics platform. AMIA Annu Symp Proc 2009; 391-5.

11. Harris PA, Taylor R, Thielke R, et al. Research electronic data capture (REDCap)--a metadata-driven methodology and workflow process for providing translational research informatics support. J Biomed Inform 2009; 42:377-81.

12. Rosenfeld RM, Andes $D$, Bhattacharyya $N$ et al. Clinical practice guideline on adult sinusitis. Otolaryngol Head Neck Surg 2007; 137:365-77.

13. Lund VJ, Kennedy DW. Staging for rhinosinusitis. Otolaryngol Head Neck Surg 1997; 117:S35-40.

14. Metson R, Gliklich RE, Stankiewicz JA, et al. Comparison of sinus computed tomography staging systems. Otolaryngol Head Neck Surg 1997; 117:372-9.

15. Hopkins C, Gillett S, Black R, Lund VJ, Browne JP. Psychometric validity of the 22-item Sinonasal Outcome Test. Clin Otolaryngol 2009; 34:447-54.

16. Chester AC. Symptom outcomes following endoscopic sinus surgery. Curr Opin Otolaryngol Head Neck Surg 2009; 17:50-8.

17. DeConde AS, Mace JC, Alt JA, et al. Longitudinal improvement and stability of the SNOT-22 survey in the evaluation of surgical management for chronic rhinosinusitis. Int Forum Allergy Rhinol 2015; 5:233-9.

18. Smith TL, Kern R, Palmer JN, et al. Medical therapy vs surgery for chronic rhinosinusitis: a prospective, multi-institutional study with 1 -year follow-up. Int Forum Allergy Rhinol 2013; 3:4-9.

19. Macdonald Kl, Gipsman A, Magit A, et al. Endoscopic sinus surgery in patients with cystic fibrosis: a systematic review and meta-analysis of pulmonary function. Rhinology 2012; 50:360-9.

20. Yip J, Yao CM, and Lee JM. State of the art: a systematic review of the surgical management of aspirin exacerbated respiratory disease. Am J Rhinol Allergy 2014; 28:493-501.

21. Caulley L, Lasso A, Rudmik L, Kitty SJ. Pretreatment SNOT-22 Score Predicts Response to Endoscopic Polypectomy in Clinic (EPIC) Our experience in 30 adults. Clin Otolaryngol. 2017:42(3):732-734

22. Bassiouni A, Chen PG, Naidoo Y, Wormwald PJ. Clinical significance of middle turbinate lateralization after endoscopic sinus surgery. Laryngoscope 2015; 125:36-41.

23. Becker SS, Han JK, Nguyen TA, Gross CW. Initial surgical treatment for chronic frontal sinusitis: a pilot study. Ann Otol Rhinol Laryngol 2007; 116:286-9.

24. Gosepath J, Pogodsky T, and Mann WJ. Characteristics of recurrent chronic rhinosinusitis after previous surgical therapy. Acta Otolaryngol 2008; 128:778-84

25. Bhattacharyya N. Computed tomographic staging and the fate of the dependent sinuses in revision endoscopic sinus surgery. Arch Otolaryngol Head Neck Surg 1999; 125:994-9

26. Anzai Y, Weymuller EA Jr. Yueh B, Maronian $\mathrm{N}$, Jarvik JG. The impact of sinus computed tomography on treatment decisions for chronic sinusitis. Arch Otolaryngol Head Neck Surg 2004; 130:423-8.

27. Ryan WR, Ramachandra T, Hwang PH.
Correlations between symptoms, nasal endoscopy, and in-office computed tomography in post-surgical chronic rhinosinusitis patients. Laryngoscope 2011; 121:674-8.

28. Tarabichi M. Characteristics of sinus-related pain. Otolaryngol Head Neck Surg 2000; 122:842-7.

29. Bhattacharyya N. The completely opacified frontal or sphenoid sinus: a marker of more severe disease in chronic rhinosinusitis? Laryngoscope 2005; 115:2123-6.

30. Myller J, Dastidar P, Torkkeli T, Rautiainen M, Toppila-Salmi S. Computed tomography findings after endoscopic sinus surgery with preserving or enlarging maxillary sinus ostium surgery. Rhinology 2011; 49:438-44.

Peter H. Hwang, MD

Professor and Vice Chair

Division of Rhinology and Endosco-

pic Skull Base Surgery

Department of Otolaryngology

Head and Neck Surgery

Stanford University School of Medi-

cine

801 Welch Road

Stanford, CA 94305

USA

Tel: +1-650-723-5651

E-mail: hwangph@stanford.edu 